\title{
Facilitated positional release
}

\author{
STANLEY SCHIOWITZ, DO
}

The facilitated positional release techniques described here introduce motion into the direction of freedom of movement. Achievement of neutral position is made easier by modifying sagittal posture, and a facilitating force is then applied. The treatment is directed at normalizing tissue texture changes but can be modified to influence the deep muscles involved in joint mobility. This modality is easily applied, nontraumatic, and efficient.

The mnemonic A-R-T is often used to describe the criteria for the diagnosis of spinal somatic dysfunction. The letters stand for Asymmetry of vertebral position, Restriction of motion of functional spinal units, and Tissue texture changes. The latter include changes in texture of fascia, muscles, subcutaneous tissues and skin. ${ }^{1}$

One of the goals of manipulation is to reverse these asymmetries, restrictions, and tissue changes. Stoddard ${ }^{2}$ stated that no matter what presenting symptoms the patient has, the physician should examine the entire spine for specific areas of reduced mobility and apply osteopathic manipulative treatment at these sites.

Dr Schiowitz is assitant dean, Office for Clinical Affairs, New York College of Osteopathic Medicine of New York Institute of Technology, Old Westbury, NY.

Reprint requests to Stanley Schiowitz, DO, Office for Clinical Affairs, New York College of Osteopathic Medicine of New York Institute of Technology, Bldg 2, Rm 363, Northern Blvd \& Glen Cove Rd, Old Westbury, NY 11568.
Direct techniques are used to create motion directly through restrictive barriers. Indirect techniques, ${ }^{3}$ such as functional ${ }^{4}$ or counterstrain $^{5}$ methods, use positional placement away from the restrictive barriers (that is, into the direction of freedom of motion with the development of reflex release patterns).

The procedures described here use the following: (1) indirect technique; (2) a modification of the sagittal posture to try to approach neutral position as defined by Fryette, ${ }^{6}$ wherein a spinal area is balanced between flexion and extension; and (3) an added force that facilitates an immediate release of tissue tension or restriction of joint motion, or both. When the procedure is properly performed, the patient will report immediate relief of point tenderness with restoration of function.

If the applied technique fails to achieve complete normalization, it can be repeated or other methods of treatment can be immediately applied.

A possible neurophysiologic explanation of how and why this type of therapy is effective was first suggested by Korr, ${ }^{7}$ when he wrote that the behavior of the "lesioned" segment suggests turned up "gain," or gamma motoneuron activity, of that segment. Bailey ${ }^{8}$ subsequently proposed that an inappropriate high gainset of the muscle spindle results in changes characteristic of somatic dysfunction.

The techniques described, by placing the region into its "neutral" position, release the facets and allow for rapidity and ease of response to applied motion and force. Application of the facilitating force has an immediate effect on the muscle spindle-gamma loop. This allows the extrafusal muscle fibers to lengthen to 
their normal relaxed state. Carew,${ }^{9}$ in discussing the feedback mechanism of the muscle spindle stretch reflex, stated that shortening of the muscle more than intended causes a decrease in spindle output, lowering Ia excitatory input to the motoneuron and less firing. As a result, muscle tension will be reduced. By placing the tissue or articulation into its specific position of freedom of movement, the physician is "fine tuning" this muscle spindlegamma loop response, making it specific for the muscle fibers involved in the somatic dysfunction being treated.

The response to this treatment is in the muscle tissue. The joint motion will normalize if its mobility was impaired by muscle hypertonicity. If joint motion asymmetry is caused by other factors such as meniscoid or synovial impingement or degenerative arthritis, then mobility will remain restricted.

The treatment procedures described in this article are divided into two kinds. One is directed at the normalization of palpable tissue texture, whereas the other is modified to influence deep muscle involved in joint mobility.

It is sometimes difficult for the physician to make a clear diagnostic distinction as to which is the primary somatic dysfunction, changes in tissue texture or motion restriction. If in doubt, it is recommended that the palpable tissue changes be treated first. If motion restriction persists, then a technique designed to normalize the deep muscles involved in the specific joint motion restriction is applied.

The methods used by the physician in making a diagnosis may consist of "skin rolling" as described by Mennell, ${ }^{10}$ testing for thoracic/ lumbar "rotoscoliosis" in the method of Mitchell, Moran, and Pruzzo, ${ }^{11}$ palpatory motion testing as described by Johnston, ${ }^{12}$ or any of the other direct or indirect tissue or motion testing procedures described in the literature, depending on the physician's expertise. It is the ultimate diagnosis, and not how it was made, that dictates the therapeutic approach.

A general description of the application of these techniques follows: The first step taken in these techniques should be the modification of the sagittal posture to create a flattening of the anteroposterior spinal curve in the region to be treated. Thus, a mild reduction of the normal cervical and lumbar lordosis or the thoracic kyphosis is established. The facilitating forces that are added consist of compression, torsion, or a combination of the two. It is important that the dysfunctional tissue or articulation to be treated is placed into positions that move freely or are pain free, or both. ${ }^{13}$

\section{Tissue texture changes}

The physician places the patient in a relaxed position. Next, the physician flattens the anteroposterior spinal curve of the area to be treated. The tissue is placed into its position of ease of motion (that is, muscle shortened and softened). The facilitating force compression, torsion, or a combination of the two is applied. The position is held for 3 to 4 seconds, then released and the condition is reevaluated. The placement of tissue into its position of ease and application of the facilitating force may be done in reverse order.

\section{Intervertebral motion restrictions}

The procedures are the same as the tissue changes, except that the physician places the vertebra into its planes of freedom of motion. For example, a somatic dysfunction diagnosed as the third cervical vertebra moving freely on the fourth cervical vertebra into the motions of extension, sidebending to the right, and rotation to the right is treated by placing the third cervical vertebra into a position of extension, right lateral flexion, and right rotation with respect to the fourth cervical vertebra.

\section{Methods for cervical region}

An area of change in tissue texture found posterior to the right articular facet of the third cervical vertebra can be treated as follows: The patient should be in the supine position with the physician sitting at the head of the table. A pillow is placed on the physician's lap. The patient is requested to move up the table toward the physician until the patient's head and neck are off the table, resting, relaxed and comfortable, on a pillow.

(continued on page 151) 
Evidence directly linking elevated cholesterol and CAD made you take a closer look at lipid levels

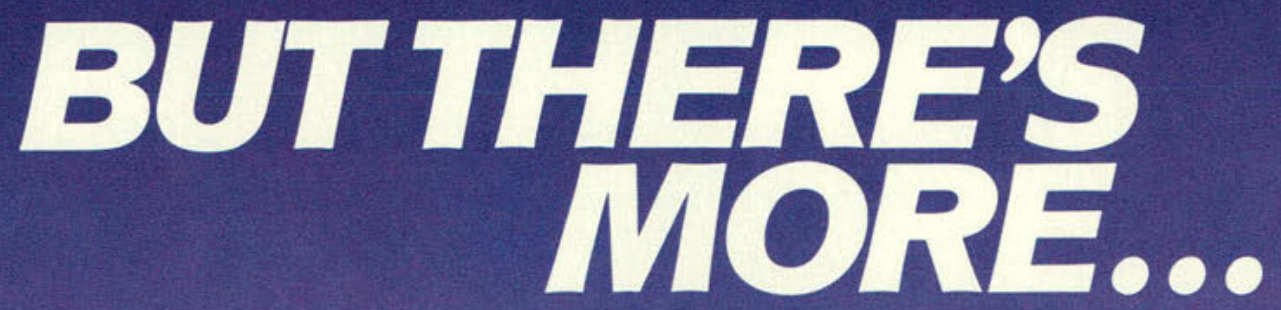


Lorelco is indicated for the reduction of elevated serum cholesterol in patients with primary hypercholesterolemia, as an adjunct to diet.
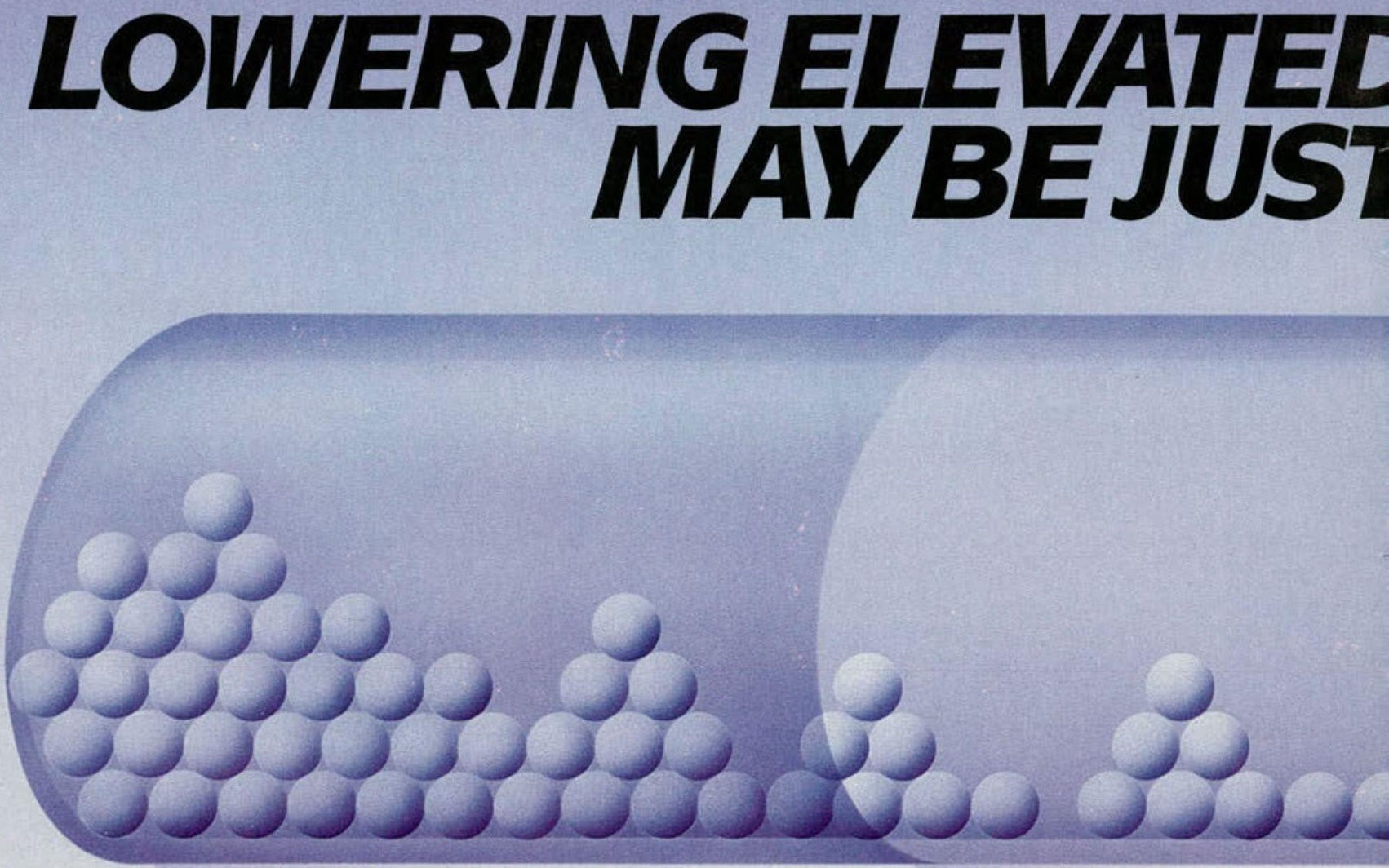

Lorelco (probucol) offers proven longterm cholesterollowering efficacy*

- Reduces total cholesterol by up to $27 \%^{1.2}$

- Maintains lower levels, as proven over a 12-year period ${ }^{3}$

- Provides the assurance of extensive clinical experience

* The effect of probucol-induced reduction of serum cholesterol or triglyceride levels or reduction of $\mathrm{HDL}$-cholesterol levels on morbidity or mortality due to coronary heart disease has not been established.

References: 1. SAfr Med J 1982:62:7-11. 2. Arch Intern Med 1981:141:1428-1432 3. Data on file, MERRELL DOW PHARMACEUTICALSINC, Cincinnati, Ohio 45242 9553. 4. Am J Cardiol 1988:62:31B-36B. 5. Atherosclerosis 1986:62:209-217. 6. Proc Natl Acad Sci USA 1987:84:5928-5931, 7. J Clin Invest 1986:77:641-644. 8. Proc Natt Acad Sci USA 1987:84:7725-7729. 9. Am J Cardiol 1988:62:6B-12B. 10. Am J Cardiol 1986:57:16 H-21H. 11. Am J Cardiol 1988:62:57B-59B. 12. Am Cardiol 1988;62:66B-72B. 13. Am J Cardiol 1988:62:73B-76B. 14. Am J Cardiol 1988:62:52B-568.

\section{Lorelco affects cholesterol in unique urays ${ }^{\dagger}$}

- Inhibits the oxidative modification of $L D L^{4-10}$

- Enhances HDL-mediated reverse cholesterol transport $\neq \S$

†Unique modes of action suggested by rece in vitro studies and human and/or animal in vivo data; clinical significance, however, is $r$. yet established.

$\ddagger$ These effects of probucol on LDL-(increase ir abolic rate) and HDL-cholesterol may be linkec observed increased excretion of fecal bile acids final metabolic pathway for elimination of chole from the body. (See Clinical Pharmacology.)

§The probable benefits obtained from $L D L$-chole reduction must be weighed against the possible $r$ of a reduction in HDL-cholesterol when assessing response of each patient receiving treatment with Lorelco. If satisfactory lipid alteration is not achieve the drug should be discontinued. (See Precautions. 


\section{LEVELS \\ THE BEGINNING}

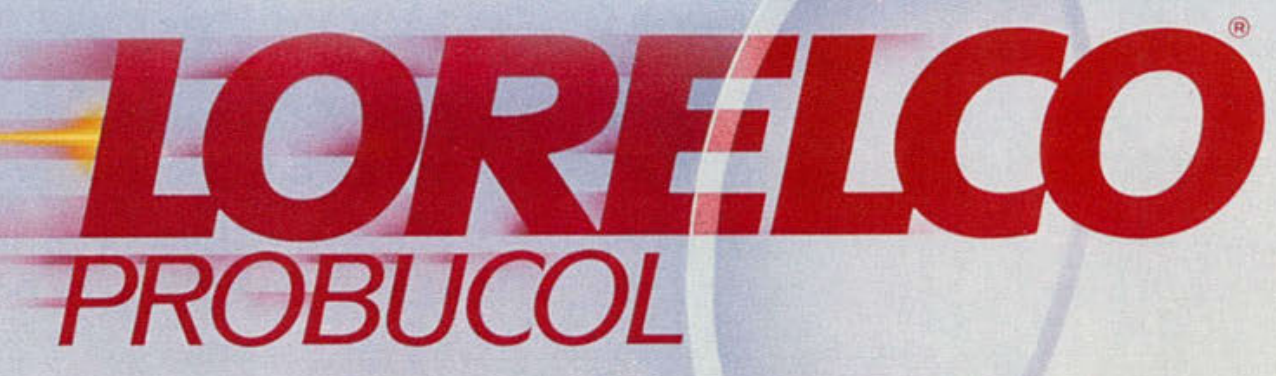

\section{Lorelco provides important patient compliance benefits}

- Convenient dosage-just one $500 \mathrm{mg}$ tablet b.i.d. with meals - Well tolerated - the most frequent side effect is loose stools, which occurs in about one in ten patients and generally subsides during continued therapy. See Prescribing Information for full discussion of side effects.

- Economical-can offer significant cost savings vs most other cholesterol-lowering therapies

Lorelco is not an innocuous drug and strict attention should be paid to the Indications, Contraindications, Warnings, and Precautions sections of Prescribing Information. 


\section{Lorelco ${ }^{\circledR}$ Tablets (probucol) \\ CAUTION: Federal law prohibits dispensing without prescription.}

DESCRIPTION: Lorelco (probucol) film-coated tablets for oral administration contain $250 \mathrm{mg}$ or $500 \mathrm{mg}$ of probucol per

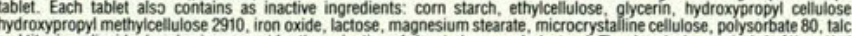

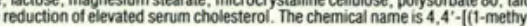
ethyl)phenol], Its chemical structure does no resemble that of any other available cho-

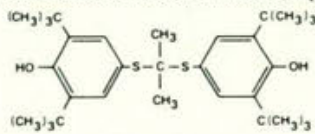

CLINICAL PHARMACOLOGY: Lorelco lowers total serum cholesterol and has relatively little effect on serum triglycerides.

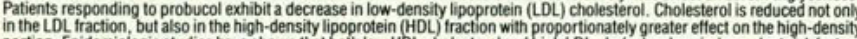

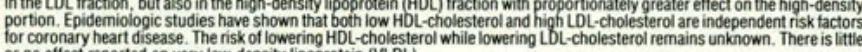
or no effect reported on very low-density lipoprotein (VLDL).

Studies on the mode of action of Lorelco indicate that it increases the fractional rate of LDL catabolism. This effect may be linked to the observed increased excretion of fecal bile acids, a final metabolic pathway for the elimination of cholesterol from the body. Lorelco also exhibits inhibition of early stages of cholesterol biosynthesis and slight inhibition of absorption of dietary
cholesterol. There is no increase in the cyclic precursors of cholestero,, namely desmosterol and 7 -dehydrocholesterol. On this

Absorption of Lorelco from the gastrointestinal tract is limited and variable. When it is administered with food, peak blood levels are higher and less variable. With continuous administration in a dosage of $500 \mathrm{mg}$ b.i.d. . the blood levels of an individual
gradually increase over the first three to four months and thereatter remain fairly constant. in 116 patients treated with Lorelco
for periods of three months to one year, the mean blood level was $23.6 \pm 17.2 \mathrm{mco} / \mathrm{mL}( \pm S .0$ ) ranging to $78.3 \mathrm{mcg} / \mathrm{mL}$ Levels observed after seven years of treatment in 40 patients yielded an average value of $21.5 \pm 16.5 \mathrm{mcg} / \mathrm{m}$ ( $1.78 .3 \mathrm{mcg} / \mathrm{mL}$. to $62.0 \mathrm{mcg} / \mathrm{mL}$. In a separate study in eight patients, blood levels averaged $19.0 \mathrm{mcg} / \mathrm{mL}$ a t the end of 12 months of the In December 1984, a National Institutes of Health Consensus Development Conference Panel1 concluded that lowering definitely coronary heart disease. The effect of probucol-induced reduction of serum cholesterol or triglyceride levels, or reduction of HDL-cholesterol levels on morbidity or mortality due to coronary heart disease has not been established. INDICATIONS AND USAGE: Serious animal toxicity has been encountered with probucol. See WARNINGS and ANIM PHARMACOLOGY AND TOXICOLLGY sections. Probucol is not an innocuous drug and strict attention should be paid to the Drug therapy should not be used for the routine treatment of elevated blood lipids for the prevention of coronary heart disease. Dietary therapy specific for the type of hyperlipidemia is the initial treatment of choice. Excoss body weight may be an importan disease such as hypothyroidism or diabetes mellitus should be looked for and adequately treated. The use of drugs should be considered only when reasonable attempts have been made to obtain satisfactory results with nondrug methods. If the d-its
ultimately is to use drugs, the patient should be instructed that this does not reduce the importance of adhering to diet. The selection of patients for cholesterol-lowering drug therapy should take into account other important coronary risk factors such as smoking. hypertension, and diabetes mellitus. Consideration should be given to the efficacy, safety, and compliance
tactors for each of the cholesterol-lowering drugs prior to selecting the one most appropriate for an individual patient. Lorelco may be indicated for the reduction of elevated serum cholesterol in patients with primary hypercholesterolemia (Types control of dasetes mellitus. Le whos control of diabetes mellitus. Lorelco may be useful to lower elevated LDL-cholesterol that occurs in those patients with
combined hypercholesterolemia and hypertriglyceridemia (Type IIt) due to elevation of both $L D L$ and VLDL, but it is not
indicated where indicated where hypertriglyceridemia is the abnormality of most concern. After establishing that the elevation in serum total cholesterol represents a primary lipid disorder, it should be determined that patients being considered tor treatment witu Lorelco
have an elevated $\mathrm{LDL}$-cholesterol as the cause for an elevated total serum cholesterol. This may be particularly reievant for patients with elevated triglycerides or with markedly elevated $\mathrm{HDL}$-cholesterol values, where non-CDL fractions may contribute be estimated according to the following equation?

$$
\text { LL -cholesterol }=\text { Total cholesterol }-[(0.16 \times \text { triglycerides })+\text { HDL-cholesterol }]
$$

When total triglycerides are greater than $400 \mathrm{mg} / \mathrm{dL}$, this equation is less accurate. In such patients, LDL-cholesterol may be It is not always possible to predict from the lipoprotein type or other factors which patients will exhibit tavorable results. Lipid The effect of probucol-induced reduction of serum cholesterol or triglyceride levels, or reduction of HDL-cholesterol levels on CONTRAINDICATIONS: (See also WARNINGS and PRECAUTIONS.) Lorelco is contraindicated in patients who are known to have a hypersensitivity to it. Lorelco is contraindicated in patients with evidence of recent or progressive myocardial damage of findings suggestive of serious ventricular arrinythmias or with unexplained syncope or syncope of cardiovascular origin.
Lorelco is contraindicated in patients with an abnormally long QT interval.

WARNINGS: SERIOUS ANIMAL TOXICITY HAS BEEN ENCOUNTERED WITH PROBUCOL IN RHESUS MONKEYS FED AN

Prolongation of the QT interval can occur in patients on Lorelco. Serious arrhythmias have been seen in association with an abnormally long QT interval in patients on Lorelco alone and in patients on Lorelco

1. Patients should be advised to adhere to a low cholesterol, low fat diet at the start of treatment with Lorelco and throughout the

2. An ECG should be done prior to starting treatment and repeated at appropriate intervals during treatment. If an abnormally
long QT interval is observed, the possible benefits and risks should be carefully considered before making a decision to long aT interval is observed, the possible benefits and risks should be carefully considered before making a decision to
continue Lorelco. Lorelco therapy should be discontinued or not started
persistently more than one of the values listed below:

QT Interval in $\sec (15 \%$ above the

\begin{tabular}{ccc}
\multirow{2}{*}{$\begin{array}{c}\text { Observed Heart Rate } \\
\text { (beats/min) }\end{array}$} & \multicolumn{2}{c}{$\begin{array}{c}\text { OT Interval in sec (15\% above the } \\
\text { upper limit of normal) }\end{array}$} \\
\cline { 2 - 3 } 40 & Males & Females \\
\hline 50 & 0.56 & 0.58 \\
60 & 0.52 & 0.53 \\
70 & 0.49 & 0.50 \\
80 & 0.45 & 0.47 \\
86 & 0.43 & 0.44 \\
92 & 0.42 & 0.43 \\
100 & 0.40 & 0.41 \\
109 & 0.39 & 0.30 \\
120 & 0.37 & 0.36 \\
133 & 0.36 & 0.35
\end{tabular}

- Values calculated from Burch GE, Winsor T. A primer of electrocardiography. Philadelphia, PA: Lea and Febiger; 1958; p.
272 (Table 6).

3. Patients developing unexplained syncope or syncope of cardiovascular origin should have Lorelco therapy discontinued and
should have ECG surveilance.

4. Drugs that prolong the $\mathrm{QT}$ interval are more likely to be associated with ventricular tachycardia after:

a. An increase in the dose of the drug.
b. Addition of a second drug that prolongs the QT interval (including tricyclic antidepressants, class I and III antiarrtythmics.
and phenothiazines).

c. Hypokalemia or hypomagnesemia.

c. Hypokalemia or hypomagnesemia.
d. Severe bradycardia due to intrinsic heart disease or drug effects on the atrial rate (beta-blockers) or AV block (digoxin).
e. Development of recent or acute myocardial infarction, ischemia, or inflammation. The use of Lorelco in patients receiving any of these drugs should be based on the conclusion that alternate methods of hypocholesterolemic therapy are either ineffective or not tolerated, and the poten

The following conditions should be resolved or corrected prior to initiation of therapy with Lorelco:

a. Hypokalemia

c. Severe bradycardia due to intrinsic heart disease or drug effects on the atrial rate (beta-blockers) or AV block (digoxin). PRECAUTIONS

General: Before instituting therapy with Lorelco, adeguate baseline studies should be performed to determine that the patient
has persistently elevated total and LDL-cholesterol levels representing a primary lipid disorder, and that the increased has persistently elevated total and LDL-cholesterol levels representing a primary lipid disorder, and that the increased cholesterol is not due to secondary conditions such as hypothyroidism, poorly controlied diabetes mellitus, obstructive liver
disease, nephrotic syndrome, or dysproteinemias. Serum lipid levels, including HDL - -cholesterol, should be determined after an
overnight tast tefore treatment, overnight fast before treatment, during an adequate trial of diet and weight reduction therapy prior to addition of drug therapy.
and periodically during combined dief and drug therapy, including assessment during the first several months of drug treatment
A favorable trend in lipid levels should be evident during the first three to four months of administration of Lorelco, and iil satistactory lipid alteration is not achieved, the drug should be discontinued. Lorelco lowers serum total and LDL-cholesterol
and also lowers $\mathrm{HDL}$-cholesterol in most and also lowers $\mathrm{HDL}$-cholesterol in most patients with elevated $\mathrm{LDL}$-cholesterol. Epidermiologic studies within hypercoronary heart disease (see CLINICAL PHARMACOLOGY). Human studies which will attempt to confirm or deny the hypothesis
that drug-induced alteration in HDL-cholesterol affects cardiovascular risk are currently under evaluation. It is not known
whether Lorelco-induced reduction of serum HDL-cholesterol will affect cardiovascular risk since no long-term controlled whether Lorelco-induced reduction of serum HDL-cholesterol will affect cardiovascular risk since no long-term, controlled been pertormed. The probable benefits obtained from LDL-cholesterol reduction must be weighed against the possible risk of a
reduction in $\mathrm{HDL}$-cholesterol when assessing the response of each patient receiving Lorelco treatment. If satistactory lipid
alteration is not achieved, the drug should be discontinued.
Information for Patients: The patient should be instructed to adhere to a prudent diet. Females should be cautioned against
becoming pregnant for at least six months after discontinuing Lorelco and should not breast-leed their intants during therapy

Laboratory Tests: The physician should schedule periodic blood lipid determinations and periodic ECGs. (See WARNINGS.) Elevations of the serum transaminases (SGOT, SGPT), bilirubin, alkaline phosphatase, creatine phosphokinase, uric acid, blood
urea nitrogen, and blood glucose above the normal range were observed on one or more occasions in various patients treated urea nitrogen, and blood glucose above the normal range were observed on one or more occasions in various patients treated
with Lorelco. Most often these were transient and ror could have been related to the patient's clinical state or other modes of these are drug related cannot be excluded. In controlled trials, the incidence of abnormal laboratory values was no higher in the patients treated with Lorelco than in the patients who received placebo. If abnormal laboratory tests persist or worsen, if clinical
signs consistent with the abnormal laboratory tests develop, or if systemic manifestations occur, Lorelco should be discon-
tinued. Drug Interactions: The addition of clofibrate to Lorelco is not recommended, since the lowering effect on mean serum levels
of either LDL or total cholesterol is generally not significantly additive and, in some patients, there may be a pronounced Neither oral hypoglycemic agents nor oral anticoagulants

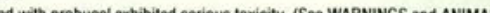
Monkeys fed a high fat, high cholesterol diet admixed with probucol exhibited serious toxicity. (See WARNINGS and ANIMAL
PHARMACOLOGY ANO TOXIICLOGY sections.) Prolongation of the OT interval can occur in patients on Lorelco and serious arrtythmias have been seen in association with an abnormally long Of interval in patients on Lorelco. The addition of a second increase the risk of serious arrmythmia. (See CONIRAINDICATIONS AND WARNINGS.)

Carcinogenesis, Mutagenesis, Impairment of Fertility

In chronic studies, of two years 'duration in rats, no toxicity or carcinogenicity was observed. These results are consistent with Pregnancy
Teralogenic Effects

Pregnancy - Category B: Reproduction studies have been performed in rats and rabbits at doses up to 50 times the human dose, and have revealed no evidence of impaired fertility or harm to the fetus due to probucol. There are, however, no adequate response, this drug should be used during pregnancy only if clearry needed Furthermore, if a patient wishes to become
pregnant, it is recommended that the drug be withdrawn and birth control procedures be used for at least six months because of persistence of the drug in the body for prolonged periods. (See CLINICAL. PHARMACOLOGY.)

Labor And Delivery: The effect of Lorelco on human labor and delivery is unknown.

Nursing Mothers: It is not known whether this drug is excreted in human milk, but it is likely, since such excretion has been Pediatric Use: Safty and effectiveness in children have not been established.

ADVERSE REACTIONS

Gastrointestinal

ols, flatulence, abdominal pain, nausea, vomiting, indigestion, gastrointestinal bleeding

prolongation of the QT interval on ECG, syncope, ventricular arrhythmias (ventricular tachycardia, torsades de pointes,

Neurologic

headache, diziness, paresthesia, insomnia, tinnitus, peripheral neuritis

Hematologic

(t)

Dermatologic
rash, prurntus, ecchymosis, petechiae, hyperhidrosis, fetid sweat

Genitourinary
impotency, nocturia

Ophthalmic

conjunctivitis, tearing, blurred vision

Endocrine
enlargement of multinodular goiter

Idiosyncrasies

Other
diminished sense of taste and smell, anorexia, angioneurotic edema

DRUG ABUSE AND DEPENDENCE: No evidence of abuse potential has been associated with Lorelco, nor is there

OVERDOSAGE: There is a single report of a 15-kg, three-year-old, male child who ingested $5 \mathrm{~g}$ of probucol. Emesis was induced by ipecac. The child remained well, apart from a briel episode of loose stools and flatulence. No specific information Treatment is symptomatic and supportive. Probucol has
animals the $L Q_{2}$ (oral) is in excess of 5 g/kg of body weight DOSAGE AND ADMINISTRATION: For adult use only. The recommended and maximal dose is $1000 \mathrm{mg}$ d
two divided doses of $500 \mathrm{mg}$ each (two $250 \mathrm{mg}$ tablets or one $500 \mathrm{mg}$ tablet) with the morning and evening meals.

HOW SUPPLIED: $250 \mathrm{mg}$ round, white, film-coated tablets imprinted with either the DOW diamond trademark over the (

$500 \mathrm{mg}$ capsule-shaped, white, film-coated tablets, marked LORELCO 500. Bottles of 100 (NDC 0068-0053-61)

Keep well closed. Store in a dry place. Avoid excessive heat. Dispense in well-closed light-resistant containers with child-

ANIMAL PHARMACOLOGY AND TOXICOLOGY: In rhesus monkeys, administration of probucol in diets containing unusually high amounts of cholesterol and saturated fat resulted in the death of tour of eight animals after several weeks.
Premonitory syncope was frequently observed and was associated with a pronounced prolongation of the $0 \mathrm{~T}$ intervals $(30$ to Premonitory syncope was frequently observed and was associated with a pronounced prolongation of the QT intervals ( 30 to
$50 \%$ longer than that observed in untreated monkeys). Serum levels of probucol greater than $20 \mathrm{mcg} / \mathrm{mL}$ were generally from control values was usually seen at $40 \mathrm{mcg} / \mathrm{mL}$ and above. Blood levels in humans receiving Lorelco average approximately
$20 \mathrm{mcg} / \mathrm{mL}$ and not uncommonly reach levels of $40 \mathrm{mcg} / \mathrm{mL}$ and higher. Rhesus monkeys fed normal (low fat) chow and receiving probucol three to thirty times the human dose equivalent achieved blood levels only one-third those of many human subjects. No adverse effects were detected in these monkeys over an eight-year period of continuous drug administration. In
another study in mesus monkeys, an atherogenic diet was ted for two years and daily treatment with probucol, separated in time another study in rhesus monkeys, an atherogenic diet was ted for two years and daily treatment with probucol, separated in time
from the atherogenic meal, was carried out during the second year. Serum probucol levels ranged 20 to $50 \mathrm{mcg} / \mathrm{mL}$ in five of ten monkeys, and less in the remaining animals. Marked prolongation of the $0 f_{i}$ interval in the ECG or syncopal behavior was never
observed over the entire one-year treatment period. Regression of gross aortic lesions comparable to that observed in a paralle group of monkeys receiving cholestyramine was seen in animals receiving probucol. it should be emphasized that both $H D L$ cholesterol and $\mathrm{DL}$-cholesterol were markedly reduced in this regression study. During the performance of a two-year chronic
study involving 32 probucol-treated dogs (beagles), there were 12 tatalities. Subsequent experiments have indicated that probucol sensitizes the canine myocardium to epinephrine, resulting in ventricular fibrillation in many dogs. Among the animal species in which probucol has been studied, the dog is pecular with respect to the
phenomenon of sudden death due to the sensitization of the myocardium to epinephrine. In contrast to findings in the dog. phienomenon of sudden death due to the sensitization of the myocardium to epinephrine.
injections of epinephrine to probucol-treated monkeys did not induce ventricular fibrillation.

In other studies, monkeys were given probucol either before and after, or only after myocardial infarction was induced by
coronary artery igation. In these studies, there was no difterence between probucol- and placebo-treated groups with respect to either survival or detailed bind quantitation of myocardiar changes (gross and histopathologic).

Probucol has shown no identifiable toxicity in mice and rats. In these animals, the LO50 (oral) is in excess of $5 \mathrm{~g} / \mathrm{kg}$ of body From studies in rats, dogs, and monkeys, it is known that probucol accumulates slowly in adipose tissue. Approximately $90 \%$ of probucol administered orally is unabsorbed. For that which is absorbed, the biliary tract is the major pathway for clearance from Myocardial injury was produced in various groups of rats by one of the following procedures: aortic coarctation, coronary as measured by survival and microscopic examination of myocardial damage.

Probucol was administered to minipigs beginning ten days betore ligation of coronary artery and continued for 60 days after surgery. Chatllenge with epinephrine at the end of 60 days tailed to induce ventricular fibrillation in any of the coronary-ligated.
probucol-treated minipigs.

CLINICAL STUDIES: In a multicenter, randomized, double-blind study, the LRC-CPPT, 'bypercholesterolemic patients
treated with an oral bile acid sequestrant (cholestyramine) and a cholesteroi-lowering diet expernenced average total and LDL. cholesterol reductions greater than those obtained in the placebo group treated with diet alone. The cumulative seven-year
incidence of the primary end point- - combined incidence of definite $C H D$ death and/or definite nonfatal myocardial infarctionincidence of the primary end point-combined incidence of definte CHD death and/or definite nontatal myocardial intarction-
was $7 \%$ in the cholestyramine group and $8.6 \%$ in the placebo group. This was a $19 \%$ reduction in risk (P less than 0.05 , singletall test) of the primary end point reflecting a $24 \%$ reduction in definite $\mathrm{CHD}$ death and a $19 \%$ reduction in nonfatal myocardia

The subjects included in the study were middle-aped men (35-59 years old) with serum cholesterol levels at least $265 \mathrm{mg} / \mathrm{dl}$ and
no previous history of heart disease. It is not clear to what extent these findings can be extrapolated to other segments of the hypercholesterolemic population not studied.

The bile acid sequestrant, cholestyramine, was used in the above trial. Caution should be exercised in extrapolating these results to Lorelco sincoit differs from cholestryramine with regard to its mode of action, spectrum of cholesterol-lowering potency,
effect on $\mathrm{HDL}$-cholesterol, and possible toxicity. The effect of probucol-induced reduction of serum cholesterol levels on
morbidity or mortality due to coronary heart disease has not been established.

REFERENCES

1. Consensus Development Panel. Lowering blood cholesterol to prevent heart disease. JAMA. 1985; 253-2080-2086. 2. Fredrickson DS, Leyy RI, Lees RS. Fat transport in lipoproteins - an integrated approach to mechanisms and disorders. 3. The Lipid Research Clinics Program. The Lipid Research Clinics coronary primary prevention trial results: 1 . Reduction in Product Information as of June, 1988

MERRELL DOWUS.A.

Subsidiary of The Dow Chemical Company

Merrell Dow U.S.A.

9-1419(L0095) MDA067R May, 1989 Printed in U.S.A 
The physician then places the pad of his or her right index finger on the area of tissue texture change to be treated (Fig 1 ). The patient's head is firmly supported by the physician's left hand, which is placed palm up, with the occiput resting in it. The physician's left hand will be used to accomplish all the subsequent maneuvers.

The physician first gently forward bends the patient's head and neck to flatten the cervical lordosis. Next, the physician gently applies axial compression on the patient's occiput toward the patient's feet, creating a vector of force through the patient's head aimed at the feet. Less than a pound of force is sufficient; it needs to be only enough to be felt at the physician's right index finger.

Maintaining this compression, the physician backward bends the patient's neck and then sidebends it up to the physician's right index finger. This should cause shortening and relaxation of the tissue being treated. This position is held for 3 seconds and then released, and the area of dysfunction is reevaluated.

In general, tissues along the posterior aspect of the spine that show texture abnormalities are shortened with backward bending and sidebending to the same side. If tissue changes are found anteriorly, then forward bending is usually required. Some muscles have a contralateral sidebending function or a rotary component, or both. Those muscles must be placed in their individual shortened positions. Careful localization of the component motions of compression, forward/backward bending, and sidebending/rotation to the area of tissue texture change will allow faster and more accurate results.

If a somatic dysfunction in the same region has been diagnosed (for example, C3 extended, sidebent right, rotated right), the treatment procedure is the same as that described for the tissue texture changes with one exception. In this situation, the specific vertebra being treated is placed into all three planes of freedom of motion.

The patient and physician positions are the same as for treating change in tissue texture. The physician's right index finger is placed at the right articular facet of the third cervical

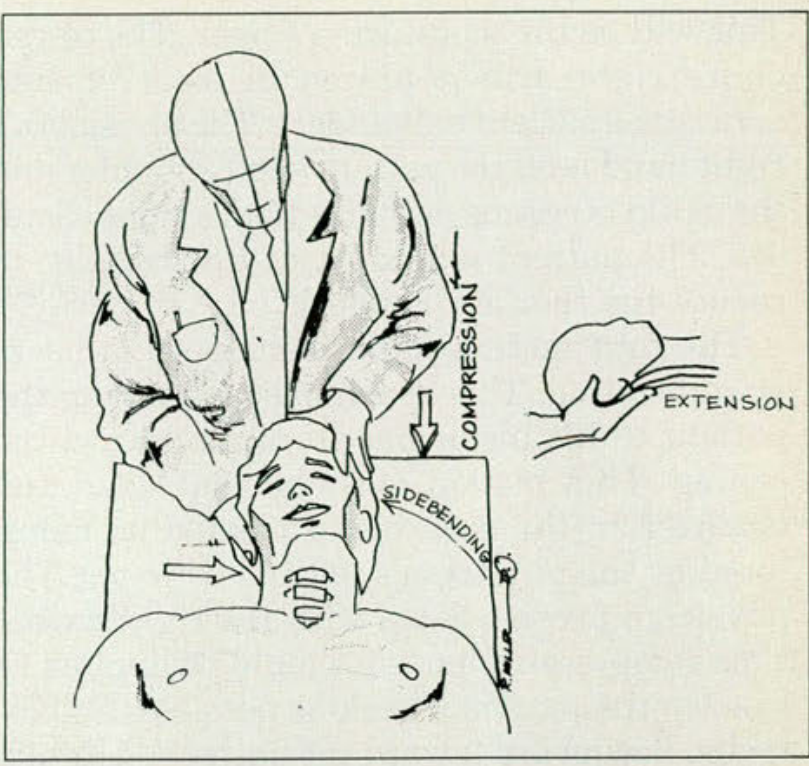

Figure 1. Treatment of tissue texture changes, posterior to the right articular facet of C3: Patient/physician position with compression and right sidebending added. B: Lateral view showing backward bending (extension) of patient's neck.

vertebra, and the physician's left hand is used to apply axial compression and accomplish extension, right lateral flexion, and right rotation of the third cervical vertebra on the fourth cervical vertebra. The physician's right index finger monitors these motions. If the technique is applied correctly, the physician's monitoring finger will feel articular facet release as the third cervical vertebra is placed into its final position. This position is held for $3 \mathrm{sec}-$ onds, released, replaced into neutral position, and reevaluated.

When applying this procedure to dysfunctions of the suboccipital area, or to the atlantooccipital articulation, the physician must remember that flexion-extension is a slight nodding motion. Also, the atlanto-occipital joint lateral flexes and rotates in opposite directions.

\section{Treatment for thoracic region}

An area of tissue tension at the sixth thoracic transverse process on the right can be treated as follows: The patient is seated. The physician stands behind and to the right of the patient. The physician places the index finger of the left hand on the site of tissue tension. 
This will be the monitoring finger. The physician's right arm is placed in front of, and across, the patient's shoulders. The physician's right hand is on the patient's left shoulder and the axilla is resting on the patient's right shoulder. The patient is told to sit up straight, to reduce the thoracic kyphosis.

The first motion to be introduced is backward bending. This is achieved by asking the patient to lift his or her sternum toward the ceiling. This motion must be controlled and localized to the area being treated as monitored by the physician's left index finger. The physician presses down with the right axilla. This compressive motion should be applied as close to the patient's neck as possible, and directed downward toward the patient's left hip. The physician monitors this motion, which creates compression and sidebending in the desired area. This position is held for 3 seconds, released, replaced into neutral position, and reevaluated.

If a somatic dysfunction is diagnosed as the sixth thoracic vertebra moving freely on the seventh thoracic vertebra into the motions of extension, sidebending to the right, and rotation to the right, the treatment procedure is modified as follows: The starting positions are as previously described with the physician's monitoring finger at the sixth articular facet on the right. The compressive force is applied straight downward toward the monitoring finger. Then right lateral flexion and rotation are introduced up to and including the sixth thoracic vertebra, so that a torsional effect is created (Fig 2). Release of the articular facet should be felt. The position is held for $3 \mathrm{sec}-$ onds, released, replaced into neutral position, and the dysfunction is reevaluated.

To apply this technique to an area that requires flexion motion, when the diagnosis is that the sixth thoracic vertebra moves freely on the seventh thoracic vertebra, into the motions of flexion, sidebending to the right, and rotation to the right, the technique would be modified as follows. The patient's position begins as before with the "sit up tall" request to reduce the thoracic kyphosis. The physician's position is as described heretofore. The compressive force is applied toward the moni-

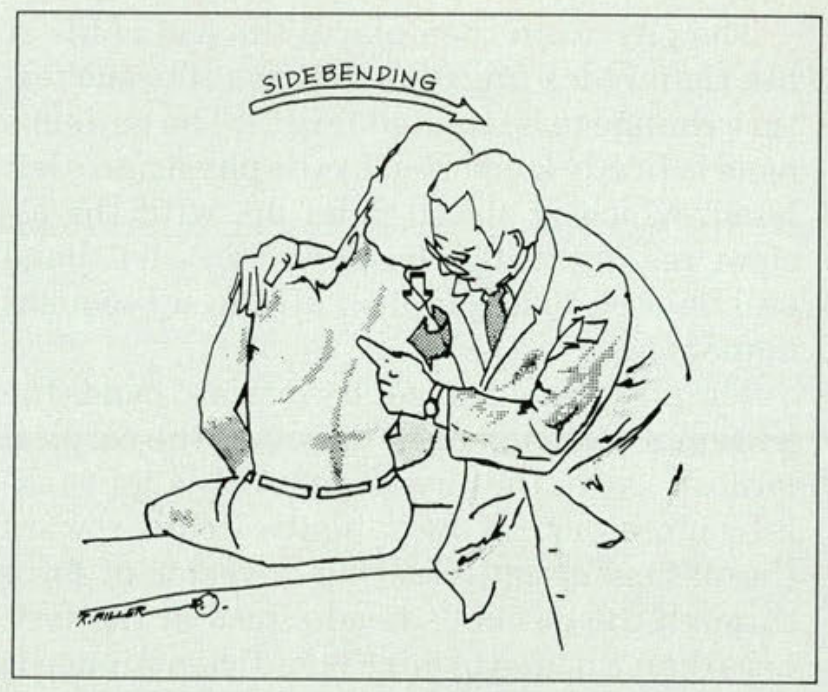

Figure 2. Physician applying a compressive force straight down, and introducing right sidebending, right rotation (torsional) motion up to the monitoring finger, for treatment of T6 moving freely on T7 into extension, sidebending to right, and rotation to right.

toring finger first. Maintaining this compression, the physician places the patient's thoracic spine into flexion, up to and including the sixth thoracic vertebra. Then, right lateral flexion and right rotation are introduced, while the facet is monitored. The position is held for 3 seconds, released, and replaced into neutral position, and the patient's condition is reevaluated.

\section{Method for lumbar region}

An area of tissue tension at the fourth lumbar transverse process on the right can be treated as follows: The patient is in the prone position with a pillow under the abdomen. The pillow should be large enough to create flattening of the lumbar lordosis. The physician stands at the right side of the table. The physician's right index finger is placed at the site of tissue tension. The physician's right knee is placed on the table lateral to the patient's right ilium.

With the left hand, the physician slides both of the patient's legs toward the right side of the table (Fig 3 ). This movement creates sidebending of the patient's lower extremities over the right knee. This procedure is continued until tissue motion is felt by the right, monitoring, index finger. 


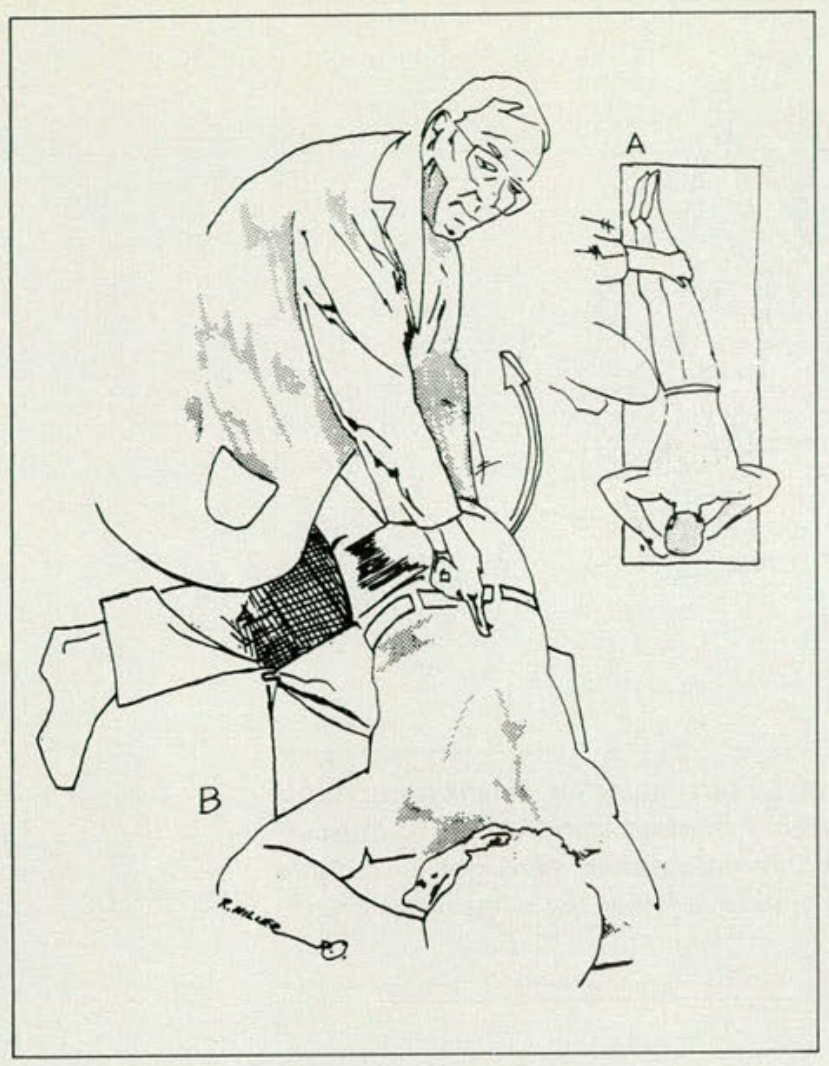

Figure 3. Treatment of tissue texture change at posterior right fourth lumbar transverse process. A: Patient position and position of physician's knee for sidebending of patient's lower extremities to right. B: Physician introduces torsional, extension/rotation motion of the left hip up to the monitoring finger.

Then the physician releases the patient's legs and places the left hand under the patient's left thigh. (This is performed with the left palm facing upward, fingers pointed toward the patient's right thigh, and the wrist in volar flexion.) The physician grasps the medial aspect of the patient's left thigh, and extends and externally rotates the thigh until motion is felt by the right monitoring finger in the area of tissue tension. A torsional motion is created up to the monitoring finger. This position is held for 3 seconds, released, and the area is reevaluated.

If the diagnosis of somatic dysfunction is that the fourth lumbar vertebra moves freely on the fifth lumbar vertebra into the motions of extension, sidebending to the right, and rotation to the right, the treatment procedure would be as follows: The patient is in the prone position with a pillow under the abdomen, and the physician stands on the right side of the table. The physician monitors the right transverse process of the lumbar vertebra with the right index finger. The physician grasps the patient's right lower leg or ankle with the left hand. The patient's right leg is brought into abduction until motion is felt at the monitoring finger, then internal rotation up to the monitoring finger is added (Fig 4A).

The patient's right leg is then pushed toward the floor until motion is felt by the physician's monitoring finger. A small pillow pad is inserted between the patient's thigh and the table to avoid creating a painful pressure area (Fig 4B). This pillow also assists in the creation of the desired fulcrum effect. The position is held until the physician feels a sudden release of joint restriction, which usually occurs in about 3 to 4 seconds. The position is released, returned to the neutral position, and the patient's condition is reevaluated.

If the somatic dysfunction is that the fourth lumbar vertebra moves freely on the fifth lumbar vertebra into the motion of flexion, sidebending to the right, and rotation to the right, the treatment would be as follow: The patient is in the prone position, with a pillow placed under the abdomen. The physician sits on a stool at the right side of the table facing the patient's head. This places the side of the physician's left thigh and knee alongside the table. The physician monitors the transverse process of L4 on the right side with one finger of the left hand.

The physician drops the patient's flexed right knee and thigh off the table over the physician's left thigh. Then the physician grasps the patient's knee with the right hand, and flexes the patient's hip until motion is felt at the monitored transverse process (Fig 5). Subsequently, the physician pushes the patient's right knee toward the table, using his or her own left knee and thigh as a fulcrum, until motion is felt at the monitored transverse process (hip adduction). Finally, with the right hand, the physician turns the patient's knee in a circular direction creating internal rotation of the hip. This position is held for 3 to 4 seconds until release of the joint restriction is felt. The position is then released, replaced 


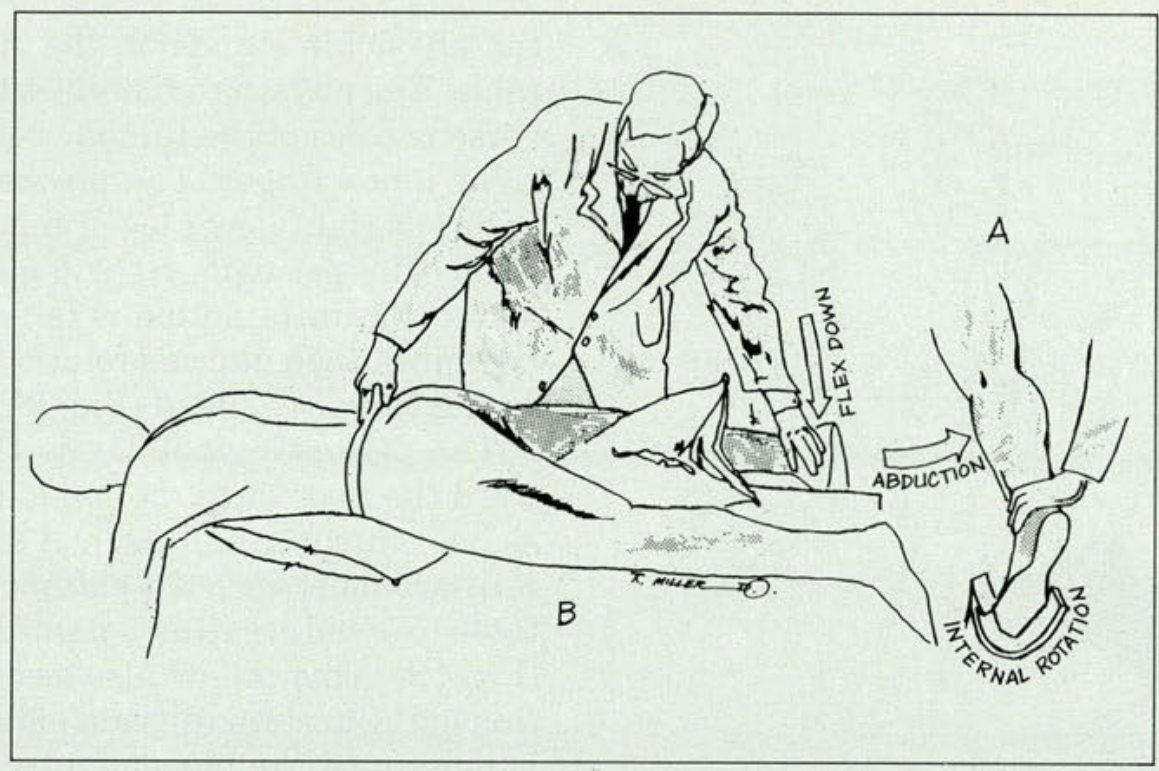

Figure 4. Treatment of L4 moving freely on L5 into extension, sidebending right, and rotation right. A: Abduction with internal rotation of right hip. B: Patient/ physician positions, maintaining abduction/internal rotation, physician introduces downward pressure on patient's foot until motion is felt at the monitoring finger.

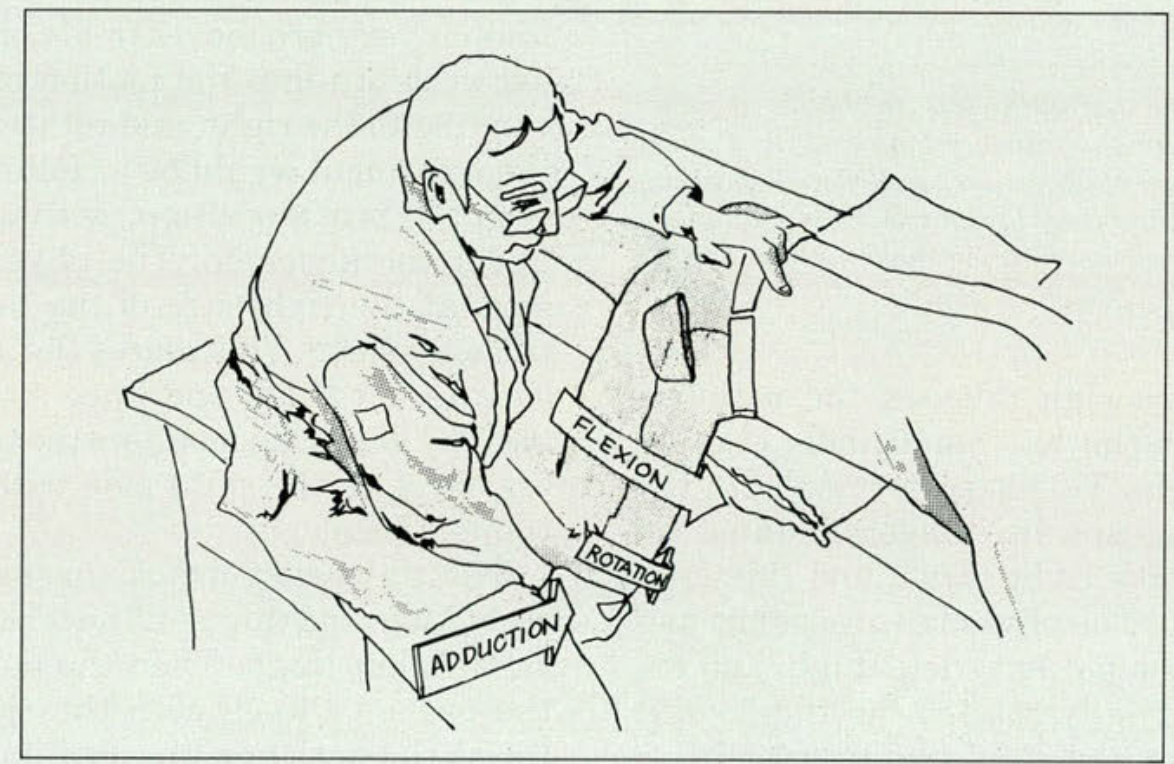

Figure 5. Introduction of flexion up to monitoring finger, and then adduction/ internal rotation of that hip until motion is felt at the monitoring finger, for treatment of L4 moving freely on L5 into flexion, sidebending right and rotation right.

into neutral position, and the patient's condition is reevaluated.

\section{Comment}

A limited number of facilitated indirect pro- cedures have been described in detail. The basic approach is applicable to other specific diagnoses. When properly used, these procedures bring about tangible, rapid resolution of somatic dysfunction. The ease with which these 
techniques can be applied and their efficaciousness should contribute to their use in daily practice by osteopathic physicians.

1. Project on Osteopathic Principles Education. Glossary of osteopathic terminology. JAOA 1981;80:552-567.

2. Stoddard A: Manual of Osteopathic Practice. New York, Harper \& Row Publishers Inc, 1969, p 38.

3. Kimberly PE: Formulating a prescription for osteopathic manipulative treatment. JAOA 1980:79:506-513.

4. Hoover HV: Functional technic. AAO Year Book 1958:47-51.

5. Jones LH: Strain and Counterstrain. Colorado Springs, Colo, American Academy of Osteopathy, 1981.

6. Fryette HH: Principles of Osteopathic Technic. Carmel, Calif, Academy of Applied Osteopathy, 1980, p 19.
7. Koor IM: Proprioceptors and somatic dysfunction. JAOA 1975;74:638-650.

8. Bailey HW: Some problems in making osteopathic spinal manipulative therapy appropriate and specific. JAOA 1976;75:486499.

9. Carew TJ: The control of reflex action, in Kandel ER, Schwartz JH (eds): Principles of Neural Science, ed 2. New York, Elsevier Science Publishing Co Inc, 1985, p 464.

10. Mennell JM: Back Pain: Diagnosis and Treatment Using Manipulative Techniques. Boston, Little Brown \& Co, 1960, p 75.

11. Mitchell FL Jr, Moran PS, Pruzzo NA: An Evaluation and Treatment Manual of Osteopathic Muscle Energy Procedures. Valley Park, Mo, Mitchell, Moran \& Pruzzo Assoc, 1979, pp 229-253.

12. Johnston WL: Segmental definition: Part 1. A focal point for diagnosis of somatic dysfunction. JAOA 1988;88:99-105.

13. Maigne $R$ : The concept of painlessness and opposite motion in spinal manipulations. Am J Phys Med 1965;44:55-69. 


\section{THE SHAPE OF THINGS TO COME FOR MILLIONS OF WOMEN}

\section{..IF OSTEOPOROSIS ISN'T TREATED}

